\title{
Post-anticoagulant D-dimer is a highly prognostic biomarker of COVID-19 mortality
}

\author{
Xiaoyu Song ${ }^{1,2}$, Jiayi $\mathrm{Ji}^{1,2}$, Boris Reva ${ }^{3}$, Himanshu Joshi $\mathbb{1}^{1}$, Anna Pamela Calinawan ${ }^{3}$, Madhu Mazumdar ${ }^{1,2}$, \\ Juan P. Wisnivesky, ${ }^{4,5}$, Emanuela Taioli ${ }^{2,6}$, Pei Wang ${ }^{3}$ and Rajwanth R. Veluswamy (iD) ${ }^{2,6,7}$
}

${ }^{1}$ Institute for Healthcare Delivery Science, Dept of Population Health Science and Policy, Icahn School of Medicine at Mount Sinai, New York, NY, USA. ${ }^{2}$ Tisch Cancer Institute, Icahn School of Medicine at Mount Sinai, New York, NY, USA. ${ }^{3}$ Dept of Genetics and Genomic Sciences and Icahn Institute for Data Science and Genomic Technology, Icahn School of Medicine at Mount Sinai, New York, NY, USA. ${ }^{4}$ Division of Pulmonary, Critical Care, and Sleep Medicine, Icahn School of Medicine at Mount Sinai, New York, NY, USA. ${ }^{5}$ Division of General Internal Medicine, Icahn School of Medicine at Mount Sinai, New York, NY, USA. ${ }^{6}$ Institute for Translational Epidemiology, Dept of Population Health Science and Policy, Icahn School of Medicine at Mount Sinai, New York, NY, USA. ${ }^{7}$ Dept of Medicine, Division of Hematology and Medical Oncology, Icahn School of Medicine at Mount Sinai, New York, NY, USA.

Corresponding author: Rajwanth R. Veluswamy (rajwanth.veluswamy@mssm.edu)

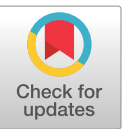

updates
Shareable abstract (@ERSpublications)

In a retrospective study of 1835 severely ill \#COVID19 patients on therapeutic anticoagulation for thromboprophylaxis during hospitalisation, post-anticoagulant D-dimer levels and trends were highly significant and independent predictors of mortality https://bit.ly/3qw4Aah

Cite this article as: Song X, Ji J, Reva B, et al. Post-anticoagulant D-dimer is a highly prognostic biomarker of COVID-19 mortality. ERJ Open Res 2021; 7: 00018-2021 [DOI: 10.1183/23120541.00018-2021].

Abstract

Copyright @The authors 2021

This version is distributed under the terms of the Creative Commons Attribution Non-Commercial Licence 4.0. For commercial reproduction rights and permissions contact permissions@ersnet.org

This article has supplementary material available from openres.ersjournals.com

Received: 8 Jan 2021 Accepted: 8 Feb 2021

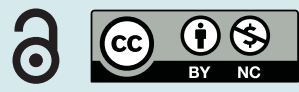

Clinical biomarkers that accurately predict mortality are needed for the effective management of patients with severe coronavirus disease 2019 (COVID-19) illness. In this study, we determine whether changes in D-dimer levels after anticoagulation are independently predictive of in-hospital mortality.

Adult patients hospitalised for severe COVID-19 who received therapeutic anticoagulation for thromboprophylaxis were identified from a large COVID-19 database of the Mount Sinai Health System in New York City (NY, USA). We studied the ability of post-anticoagulant D-dimer levels to predict inhospital mortality, while taking into consideration 65 other clinically important covariates including patient demographics, comorbidities, vital signs and several laboratory tests.

1835 adult patients with PCR-confirmed COVID-19 who received therapeutic anticoagulation during hospitalisation were included. Overall, $26 \%$ of patients died in the hospital. Significantly different inhospital mortality rates were observed in patient groups based on mean D-dimer levels and trend following anticoagulation: $49 \%$ for the high mean-increase trend group; $27 \%$ for the high-decrease group; $21 \%$ for the low-increase group; and $9 \%$ for the low-decrease group $(\mathrm{p}<0.001)$. Using penalised logistic regression models to simultaneously analyse 67 clinical variables, the high increase (adjusted odds ratios $\left(\mathrm{OR}_{\mathrm{adj}}\right)$ : 6.58, 95\% CI 3.81-11.16), low increase $\left(\mathrm{OR}_{\mathrm{adj}}: 4.06\right.$, 95\% CI 2.23-7.38) and high decrease (OR $\mathrm{adj}$ : 2.37; 95\% CI 1.37-4.09) D-dimer groups (reference: low decrease group) had the highest odds for in-hospital mortality among all clinical features.

Changes in D-dimer levels and trend following anticoagulation are highly predictive of in-hospital mortality and may help guide resource allocation and future studies of emerging treatments for severe COVID-19.

\section{Introduction}

The coronavirus disease 2019 (COVID-19) pandemic has resulted in 46 million confirmed cases and 1.2 million deaths worldwide through November, 2020 [1]. Among patients with more severe illness requiring hospitalisation, there is an urgent need for accurate clinical biomarkers to predict mortality risk in order to guide clinical decisions, allocate critical resources and inform study designs of emerging treatments. Currently, there is insufficient evidence to precisely identify patients at the highest risk of poor outcomes, and clinicians often consider a multitude of individual clinical factors (i.e., exam findings, laboratory tests) without predictive cut-off values for making treatment decisions. Therefore, quantifying the impact of 
laboratory markers used in clinical practice as prognostic biomarkers for mortality is critical for the effective management of COVID-19 patients.

D-dimer, a small protein fragment present in blood resulting from plasmin cleavage of cross-linked fibrin clots, is routinely used in clinical practice as a sensitive biomarker in the evaluation of venous thromboembolism (VTE) [2]. Recently, several studies have shown that elevated D-dimer levels at the time of hospital admission in COVID-19 patients are associated with higher mortality [3-6]. Furthermore, the use of anticoagulant therapy in hospitalised COVID-19 patients with elevated D-dimer levels resulted in a significant mortality benefit [3, 7-10]. As a consequence, many guidelines and institutional protocols have recommended therapeutic anticoagulation strategies (using intermediate or full doses) for thromboprophylaxis in patients with severe COVID-19 infection [4-6]. However, while D-dimer measurements generally are followed throughout the hospitalisation, there remains no consensus or guidance as to how changes in D-dimer levels should be interpreted following anticoagulant therapy in COVID-19 patients.

In this study, we hypothesised that changes in D-dimer levels and trends following administration of therapeutic doses of anticoagulation in patients with severe COVID-19 infections are predictive of in-hospital mortality. To determine the role of D-dimer in this setting, we leveraged a large institutional database of COVID-19 hospitalised patients from the Mount Sinai Health System (MSHS) in New York City (NY, USA), one of the initial epicentres of the COVID-19 pandemic in the USA.

Study design and methods

\section{Study cohort}

MSHS includes the Mount Sinai Hospital and seven other urban hospitals throughout New York City, serving a diverse patient population with high representation of low-income minorities. This study utilised a comprehensive COVID-19 database, which includes de-identified clinical data extracted from the electronic medical records of all patients tested for and/or diagnosed with COVID-19 within the health system from February 25, 2020, to May 31, 2020. We included all adults ( $\geqslant 18$ years of age) who were hospitalised for a new COVID-19 infection (based on RT-PCR COVID-19 assay using nasopharyngeal swabs), and were treated with therapeutic doses of anticoagulation for thromboprophylaxis. Included patients required follow up data for at least 3 days after the first anticoagulant dose and information on their hospitalisation outcome (discharged versus deceased). We then excluded patients who: 1) had high risk of bleeding from therapeutic doses of anticoagulation due to either low platelet counts $(<50000 / \mu \mathrm{L})$ or elevated international normalisation ratio (INR>1.5); and 2) were given therapeutic doses of anticoagulation or tissue plasminogen activator (TPA) for a newly diagnosed VTE as large vessel thrombosis could affect post-anticoagulant D-dimer levels.

\section{Study variables}

Patient characteristics

For each patient, we obtained baseline sociodemographic data (i.e. age, sex, self-reported race and ethnicity), smoking status, body mass index (BMI), and 18 common comorbidities (Table S1). Baseline vital signs (temperature, systolic blood pressure, diastolic blood pressure, oxygen saturation, heart rate, respiratory rate) and laboratory tests obtained within $24 \mathrm{~h}$ of admission and prior to receiving anticoagulation were collected. In cases where multiple vital signs were recorded during the first $24 \mathrm{~h}$ of admission, we used the most clinically abnormal measurement concerning for systemic inflammatory response syndrome (SIRS) [11]. A pre-processing procedure was performed to exclude laboratory tests that were missing in $>50 \%$ of patients. The remaining 35 laboratory tests that were used for analysis included complete blood count (CBC) with differential, comprehensive metabolic panel (CMP), inflammatory markers (i.e. ferritin, C-reactive protein (CRP), lactate dehydrogenase (LDH)), liver function tests and baseline D-dimer. Altogether, 65 baseline variables were considered for each patient (Table S1).

\section{Therapeutic dose of anti-coagulation treatment}

The MSHS, alongside many other high acuity hospitals, has developed a standardised protocol for anticoagulant therapy in patients requiring hospital admission for COVID-19. All patients without confirmed VTE are recommended to receive thromboprophylaxis with heparin, enoxaparin and/or apixaban using either prophylactic doses for patients without severe respiratory compromise or therapeutic doses (intermediate or full) for patients with severe respiratory compromise (i.e. respiratory rate $>24$ breaths $\cdot \mathrm{min}^{-1}$, oxygen saturation $<90 \%$, or requiring supplemental oxygen $>4 \mathrm{~L} \cdot \mathrm{min}^{-1}$ via nasal canula). Our study cohort included patients on therapeutic doses defined as: 1) heparin: $>5000$ units subcutaneous every $8 \mathrm{~h}$ in patients with $\mathrm{BMI}<40 \mathrm{~kg} \cdot \mathrm{m}^{-2}$ or $>7500$ units subcutaneous every $8 \mathrm{~h}$ in patients with $\mathrm{BMI} \geqslant 40 \mathrm{~kg} \cdot \mathrm{m}^{-2}$; 2) enoxaparin: $1 \mathrm{mg} \cdot \mathrm{kg}^{-1}$ subcutaneous every $24 \mathrm{~h}$ (intermediate dose) or $1 \mathrm{mg} \cdot \mathrm{kg}^{-1}$ subcutaneous twice daily (full dose); and 3) apixaban: $>2.5 \mathrm{mg}$ by mouth every $24 \mathrm{~h}$. 
Post-anticoagulant D-dimer values and groups

We recorded post-anticoagulant D-dimer levels as all measurements collected within the first 3 days after the administration of therapeutic doses of anticoagulation was started. This time frame was chosen after finding the incremental area under the curve (AUC) change for the predictive capability of our model was not significantly different for each additional day of D-dimer levels past day 3 (Figure S1). As the number of D-dimer measurements during this period varied dramatically (from 0 to 18 measurements) for each patient, we calculated both the mean and trend to summarise the data. The trend was defined, if at least two measurements were available, as the slope of a linear regression model characterising the dependence of the post-anticoagulant D-dimer values on the test collection time from anticoagulation.

Using $2.5 \mu \mathrm{g} \cdot \mathrm{mL}^{-1}$ as a cut-off for the post-anticoagulant D-dimer mean value, and 0 as a cutoff for the post-anticoagulant D-dimer trend, we divided patients into four groups with similar sample sizes: high increase: high mean value $\left(\geqslant 2.5 \mu \mathrm{g} \cdot \mathrm{mL}^{-1}\right)$ and increase trend (trend $\left.\geqslant 0\right)$; high decrease: high mean value and decrease trend; low increase: low mean value and increase trend; and low decrease: low mean value and decrease trend.

\section{Study end point}

The study endpoint is a binary indicator of in-hospital mortality, defined as patients who died during their admission versus patients who were discharged alive from the hospital, usually to home, nursing facility, acute/sub-acute rehab or long-term care facility.

\section{Statistical analysis}

To test the associations between baseline and post-anticoagulant D-dimer variables with in-hospital mortality, Chi-squared tests and two-sample Wilcoxon tests were used for categorical and continuous variables, respectively. Bonferroni correction for multiple testing provided $p<0.0007(=0.05 / 65)$ as the cut-off to determine significant associations with in-hospital mortality. Missing values in categorical variables were treated as a separate category, while multiple imputations were performed for missing values in numeric variables using the R package MICE [12].

Logistic regression models were employed to predict in-hospital mortality based on baseline and post-anticoagulant D-dimer levels. The predictive values were evaluated through 10 -fold cross-validation. The receiver operating characteristic (ROC) curve and the corresponding AUCs were used to assess and compare the performance of the prediction model based on baseline and post-anticoagulant D-dimer values.

We assessed whether the in-hospital mortality and the baseline characteristics of patients differed across the four D-dimer groups described above. Chi-squared tests were used for categorical variables and Kruskal-Wallis tests for continuous variables. Variables that passed 5\% significance level after Bonferroni correction were further examined for statistically significant differences in groups with high D-dimer levels (high increase and high decrease combined) versus groups with low D-dimer levels (low increase and low decrease combined); as well as in groups with increasing D-dimer versus those with decreasing D-dimer trends (high increase versus high decrease; low increase versus low decrease).

We assessed the predictivity of D-dimers for in-hospital mortality conditional on baseline characteristics of patients. To better estimate effect sizes of predictors, we randomly split the samples into discovery and validation subsets with equal sizes and performed variable selection on the discovery subset while inference of effect sizes on the validation subset to avoid post-selection inference, which results in biased estimates and confidence intervals. In the discovery subset, we utilised regularised logistic regression models with Lasso penalty [13] to select the most important predictors for in-hospital mortality from a large feature set of 67 important clinical variables (Table S1). For the variables selected by the penalised logistic regressions, we performed an ordinary logistic regression using the validation subset to estimate odds ratios and the corresponding 95\% confidence intervals. For the variables that were confirmed to be statistically significant, we calculated AUC differences between leave-one-predictor-out models and the full model in the validation subset to assess the relative importance of each predictor. Moreover, we performed parallel analyses using only baseline variables, and compared the predictive performance of these models with the above ones using post-anticoagulant D-Dimer information in the validation subset. We compared the 10-fold cross-validation prediction AUCs between the baseline model and the full model with post-anticoagulant D-dimer groups among 100 randomly selected bootstrap samples. All statistical analyses were repeated in complete case analysis among samples without missing post-anticoagulant D-dimer data, with similar results (Table S3). 
We analysed a subset of 668 patients in our cohort that had at least two post-anticoagulant fibrinogen measurements in order to determine if changes in D-Dimer levels reflected onset of disseminated intravascular coagulopathy (DIC) or if a more COVID-specific process. As fibrinogen would not be affected by anticoagulation directly, we used the mean and trend as a proxy for DIC and added these covariates in the penalised logistic regression.

\section{Results}

Baseline characteristics of study cohort

After applying the selection criteria to the COVID-19 database ( $n=65501$ patients), the final study cohort consisted of 1835 laboratory-confirmed COVID-19 positive adult patients who were hospitalised in the MSHS between February 25 and May 31, 2020 (figure 1). Among them, 470 (26\%) study patients died during hospitalisation and 1365 (74\%) were discharged alive. Patients who died during hospitalisation were generally older, had more comorbidities, presented with signs of more severe respiratory distress (higher respiratory rates and lower minimum oxygen saturation), had worse kidney function, higher levels of inflammatory markers (ferritin, CRP, LDH) and higher baseline D-dimers $(p<0.001$ for all comparisons after Bonferroni correction). Although not statistically significant, patients who died during hospitalisation also experienced a longer time between admission and the start of therapeutic doses of anticoagulation (tables 1 and S1).

\section{Post-anticoagulant D-dimer levels and COVID-19 mortality}

After beginning therapeutic doses of anticoagulation, the mean D-dimer was significantly higher for patients who died versus those who were discharged from the hospital (median (interquartile range) 3.71 $(1.98-8.05) \mu \mathrm{g} \cdot \mathrm{mL}^{-1}$ versus $1.69(0.86-3.41) \mu \mathrm{g} \cdot \mathrm{mL}^{-1}$, respectively; $\left.\mathrm{p}<0.001\right)$. The difference in mean post-anticoagulant D-dimers between discharged versus deceased groups was greater than the difference observed at baseline $\left(2.02 \mu \mathrm{g} \cdot \mathrm{mL}^{-1}\right.$ versus $0.39 \mu \mathrm{g} \cdot \mathrm{mL}^{-1}$, respectively; $\left.\mathrm{p}<0.001\right)$. An increasing trend of post-anticoagulant D-dimers was observed for patients who died in the hospital (median slope: 0.09), while a decreasing trend was seen for those who were discharged (median slope: -0.05), with a significant difference between the changes in slope $(\mathrm{p}<0.001)$ (figure $2 a)$. The predictive power for in-hospital mortality of the logistic regression model with post-anticoagulant D-dimer mean level and its trend (AUC 0.76; 95\% CI 0.74-0.78) was significantly greater than the model with the baseline D-dimer (AUC 0.59; 95\% CI 0.55-0.61). Including baseline D-dimer levels to the model based on post-anticoagulant D-dimer did not further improve the prediction (AUC 0.76; 96\% CI 0.74-0.78) (figure 2b).

By stratifying the study cohort into four similar sized groups combining high versus low post-anticoagulant D-dimer means and increasing versus decreasing trend, a significant difference was observed in the

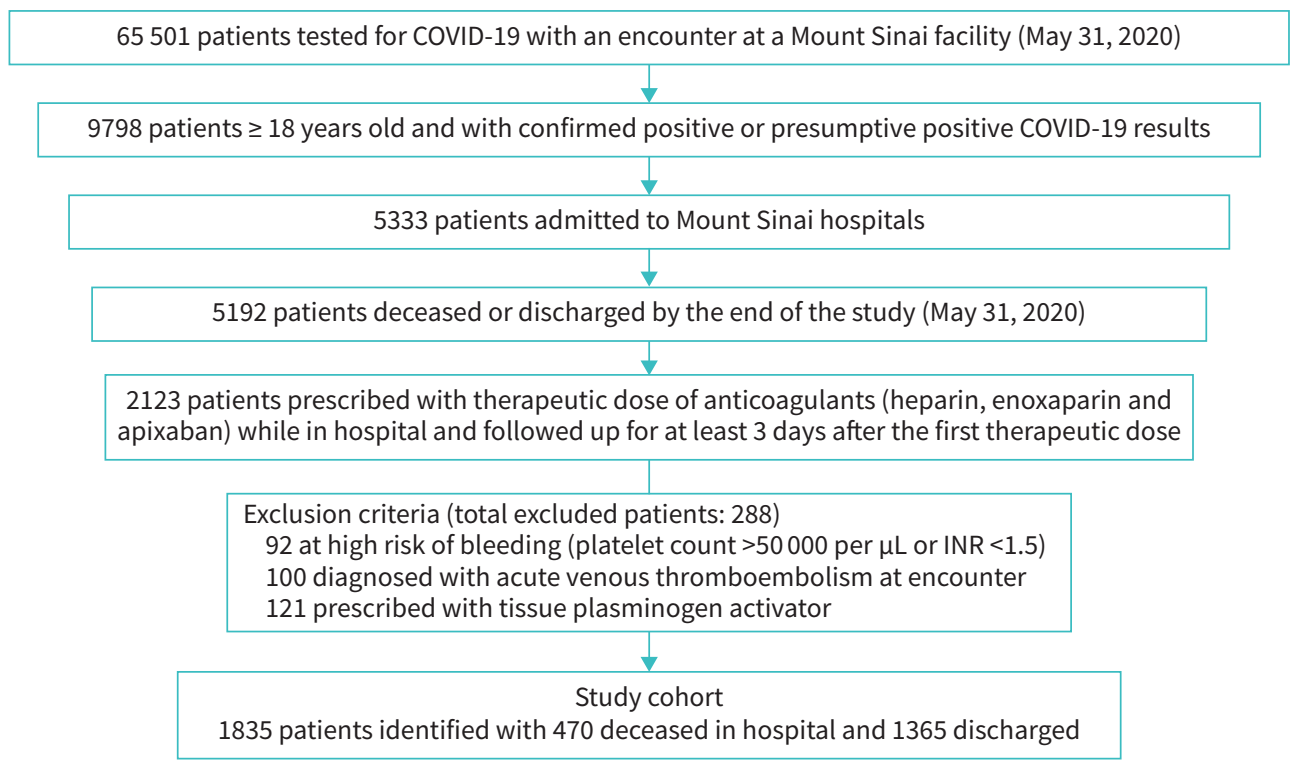

FIGURE 1 Selection of study cohort to evaluate role of post-anticoagulant D-dimer as predictive biomarker for mortality. 


\begin{tabular}{|c|c|c|c|}
\hline Characteristics & Discharged from hospital & Deceased in hospital & p-value \\
\hline Patients & 1365 & 470 & \\
\hline Age years & $65(54-75)$ & $73(65-83)$ & $<2.2 \times 10^{-16 \#}$ \\
\hline Male sex & $805(59.0)$ & $262(55.7)$ & 0.242 \\
\hline Race/ethnicity & & & 0.374 \\
\hline White & $275(20.1)$ & $111(23.6)$ & \\
\hline Black & $277(20.3)$ & $83(17.7)$ & \\
\hline Hispanic & $404(29.6)$ & $138(29.4)$ & \\
\hline Other & $236(17.3)$ & $73(15.5)$ & \\
\hline Missing & $173(12.7)$ & 65 (13.8) & \\
\hline \multicolumn{4}{|l|}{ Smoking status } \\
\hline Never smoker & $688(50.4)$ & $229(48.7)$ & \multirow{2}{*}{0.714} \\
\hline Ever smoker & $384(28.1)$ & $132(28.1)$ & \\
\hline Missing & $293(21.5)$ & $109(23.2)$ & \\
\hline \multicolumn{2}{|c|}{ Baseline comorbidities (details in Table S1) } & & $1.4 \times 10^{-08 \#}$ \\
\hline 0 & $456(33.4)$ & $89(18.9)$ & \\
\hline $1-2$ & $534(39.1)$ & $196(41.7)$ & \\
\hline$>2$ & $375(27.5)$ & $185(39.4)$ & \\
\hline BMI $\mathrm{kg} \cdot \mathrm{m}^{-2}$ & $27.55(23.88-32.12)$ & $27.46(24.32-32.48)$ & 0.331 \\
\hline \multicolumn{4}{|l|}{ Admission vital signs } \\
\hline $\begin{array}{l}\text { Systolic blood pressure } \\
\text { maximum mmHg }\end{array}$ & $143(129-159)$ & $144(129-161)$ & 0.531 \\
\hline $\begin{array}{l}\text { Diastolic blood pressure } \\
\text { maximum mmHg }\end{array}$ & $83(76-92)$ & $81(73-91)$ & 0.017 \\
\hline Heart rate maximum beats $\cdot \mathrm{min}^{-1}$ & $101.00(90.75-114.00)$ & $103.00(91.00-120.75)$ & 0.009 \\
\hline $\begin{array}{l}\text { Respiratory rate maximum } \\
\text { breaths } \mathrm{min}^{-1}\end{array}$ & $22(20-28)$ & $24(20-30)$ & $9.7 \times 10^{-06 \#}$ \\
\hline Temperature maximum ${ }^{\circ} \mathrm{F}$ & $99.8(98.6-101.3)$ & $99.6(98.6-101.1)$ & 0.064 \\
\hline Temperature maximum ${ }^{\circ} \mathrm{C}$ & $37.7(37.0-38.5)$ & $37.6(37.0-38.4)$ & \\
\hline Oxygen saturation minimum $\%$ & $93(90-95)$ & $90(84-94)$ & $3.4 \times 10^{-16 \#}$ \\
\hline \multicolumn{4}{|l|}{ Baseline laboratory tests } \\
\hline White blood cell $\times 10^{3}$ cells $\mu \mathrm{L}^{-1}$ & $7.60(5.50-10.40)$ & $8.14(5.90-12.28)$ & 0.011 \\
\hline Lymphocyte \% & $13.05(8.30-18.58)$ & $11.00(7.25-16.95)$ & 0.003 \\
\hline Neutrophil \% & $78.60(70.45-84.77)$ & $81.90(73.40-87.10)$ & $2.8 \times 10^{-05 \#}$ \\
\hline Haemoglobin $\mathrm{g} \cdot \mathrm{dL}^{-1}$ & $12.70(11.10-13.90)$ & $12.90(11.10-14.10)$ & 0.448 \\
\hline Platelet $\times 10^{3}$ platelets $\mu \mathrm{L}^{-1}$ & $221.00(167.00-293.00)$ & $187.00(147.00-244.00)$ & $1.7 \times 10^{-09 \#}$ \\
\hline Serum creatinine $\mathrm{mg} \cdot \mathrm{dL}^{-1}$ & $0.95(0.73-1.41)$ & $1.20(0.82-2.00)$ & $1.2 \times 10^{-4 \#}$ \\
\hline $\begin{array}{l}\text { Estimated glomerular filtration } \\
\text { rate } \mathrm{mL} \cdot \mathrm{min}^{-1} \cdot 1.73 \mathrm{~m}^{-2}\end{array}$ & $54.00(30.15-77.00)$ & $41.28(26.00-58.33)$ & $7.3 \times 10^{-11 \#}$ \\
\hline Alanine aminotransferase $\mathrm{U} \cdot \mathrm{L}^{-1}$ & $30.00(19.00-50.00)$ & $30.00(20.25-50.75)$ & 0.885 \\
\hline Aspartate aminotransferase $\mathrm{U} \cdot \mathrm{L}^{-1}$ & $40.00(27.00-63.50)$ & $48.00(33.00-71.75)$ & $1.7 \times 10^{-4 \#}$ \\
\hline Total bilirubin $\mathrm{mg} \cdot \mathrm{dL}^{-1}$ & $0.60(0.40-0.80)$ & $0.50(0.40-0.80)$ & 0.356 \\
\hline C-reactive protein $\mathrm{mg} \cdot \mathrm{L}^{-1}$ & $113.50(53.70-194.30)$ & $159.10(90.82-235.70)$ & $1.0 \times 10^{-09 \#}$ \\
\hline Ferritin $\mathrm{ng} \cdot \mathrm{mL}^{-1}$ & $697.00(330.00-1512.00)$ & $1038.00(480.00-2092.00)$ & $8.9 \times 10^{-05 \#}$ \\
\hline Lactate dehydrogenase $\mathrm{U} \cdot \mathrm{L}^{-1}$ & $412.00(312.00-528.50)$ & $516.50(381.25-660.75)$ & $<2.2 \times 10^{-16 \#}$ \\
\hline Baseline D-dimer $\mu \mathrm{g} \cdot \mathrm{mL}^{-1}$ & $1.37(0.79-2.46)$ & $1.76(1.08-2.92)$ & $1.6 \times 10^{-05 \#}$ \\
\hline $\begin{array}{l}\text { Days from admission to start of } \\
\text { anticoagulation }\end{array}$ & $0.55(0.20-1.68)$ & $0.67(0.24-2.13)$ & 0.017 \\
\hline
\end{tabular}

Data are presented as $\mathrm{n}, \mathrm{n}(\%)$ or median (interquartile range), unless otherwise stated. \#: Significant after multiple testing. BMI: Body mass index.

in-hospital mortality rates for patients within high increase (49\%) versus high decrease (27\%) versus low increase $(21 \%)$ versus low decrease $(9 \%)$ groups $(\mathrm{p}<0.001)$ (figure 3a). Patients with high mean post-anticoagulant D-dimer $\left(\geqslant 2.5 \mu \mathrm{g} \cdot \mathrm{mL}^{-1}\right)$ were typically older, had more comorbidities, lower oxygen saturation, higher baseline D-dimers, higher leukocyte counts with lower lymphocyte percentages, worse kidney function and higher inflammatory markers than patients with low mean post-anticoagulant D-dimer $\left(<2.5 \mu \mathrm{g} \cdot \mathrm{mL}^{-1} ; \mathrm{p}<0.001\right.$ for all comparisons) (Table S2). Among patients within the high or low mean post-anticoagulant D-dimer groups, only lower baseline D-dimer was associated with increasing D-dimer trends $(\mathrm{p}<0.001)$ (figure $3 b)$. 
a)

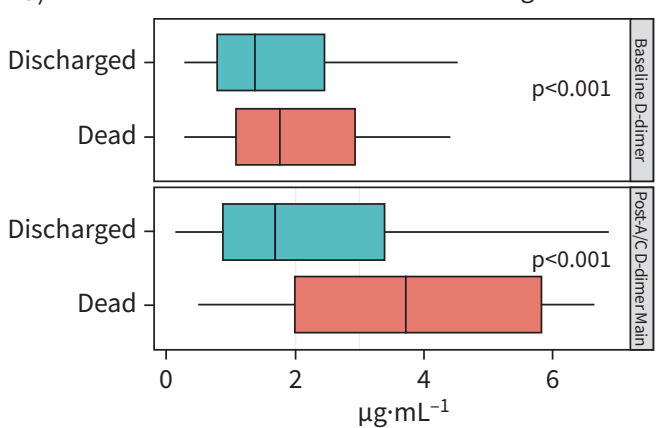

b)

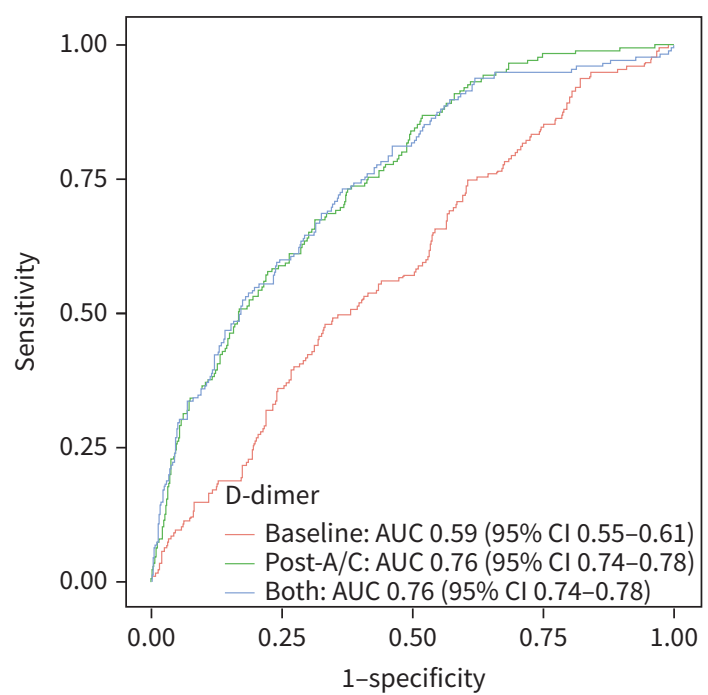

FIGURE 2 D-dimer distribution and its association with patient outcomes. a) Boxplot of baseline and post-anticoagulation (post-A/C) D-dimer values. b) receiver operating characteristic curves of prediction models with baseline D-dimer, post-anticoagulation D-dimer and both (area under the curve (AUC) $0.59,0.76$ and 0.76 , respectively).

Post-anticoagulant D-dimer groups and in-hospital mortality

Jointly modelling post-anticoagulant D-dimer groups and 65 baseline covariates with penalised logistic regressions, 12 variables were selected to be predictive of in-hospital mortality through 10 -fold cross validation based on the discovery subset. Among these, 10 variables were confirmed to be significantly associated with in-hospital mortality based on the validation subset (figure 4a). Compared with patients in the low decrease post-anticoagulant D-dimer group, patients in the high increase post-anticoagulant D-dimer group were significantly the most likely to die during hospitalisation (adjusted odds ratios $\left(\mathrm{OR}_{\mathrm{adj}}\right)$ 6.58; 95\% CI 3.81-11.16), followed by those in the low increase post-anticoagulant D-dimer group (OR $\mathrm{OR}_{\mathrm{adj}}$ 4.06; 95\% CI 2.23-7.38) and high decrease group ( $\mathrm{OR}_{\mathrm{adj}}$ 2.37; 95\% CI 1.37-4.09), after adjusting for the other pre-selected covariates. The post-anticoagulant D-dimer group was a stronger predictor of mortality than other covariates, such as acute kidney injury $\left(\mathrm{OR}_{\mathrm{adj}} 1.99\right.$; 95\% CI 1.34-2.96), or acute respiratory distress syndrome (ARDS; $\mathrm{OR}_{\text {adj }}$ 2.46; 95\% CI 1.44-4.20) at admission (figure 4a). The baseline D-dimer value was not a significant predictor of mortality and was not selected in the final model. Additionally, we found consistent results that post-anticoagulant D-dimer remained the most significant predictor of in-hospital mortality after adjusting for fibrinogen (Figure S2).

\section{Robustness of the findings}

When we evaluated the impact of individual predictors in the above model by calculating the change of the model's AUC after excluding one variable at a time, age resulted in the largest AUC change (0.041), followed by high decrease post-anticoagulant D-dimer group (0.039), high increase post-anticoagulant D-dimer group (0.024), platelet count (0.020), minimum oxygen saturation (0.020) and then low increase post-anticoagulant D-dimer group (0.019) (figure $4 \mathrm{~b}$ ). The combined effects of the four post-anticoagulant D-dimer groups had the greatest impact on model prediction (AUC change 0.054). Furthermore, when we compared the predictive powers of the above model based on post-anticoagulant D-dimer groups as well as the selected baseline variables with the predictive powers of a model based on only baseline variables, the AUCs of the post-anticoagulant D-dimer models were significantly higher than that of the baseline models based on 100 bootstrap data sets generated from the validation subset (figure 4c).

\section{Discussion}

In this retrospective study of 1835 adult patients on therapeutic doses of anticoagulation for thromboprophylaxis during admission for severe COVID-19 illness, we found high and independent predictive power of post-anticoagulant D-dimer levels for in-hospital mortality, while taking into consideration 65 other important covariates including patient demographics, comorbidities, vital signs and laboratory tests at baseline. We further identified patient-specific trajectories of D-dimers values after anticoagulant therapy, which demonstrated significant differences in mortality rates and had the greatest 


\begin{tabular}{|c|c|c|c|}
\hline Mean post-A/C D-dimer & Change over time & Patients n & Mortality rate \\
\hline \multirow{2}{*}{$\begin{array}{c}\text { High } \\
\left(\geq 2.5 \mu \mathrm{g} \cdot \mathrm{mL}^{-1}\right)\end{array}$} & $\begin{array}{c}\text { Increase } \\
\left(\geq 0 \mu \mathrm{g} \cdot \mathrm{mL}^{-1} \text { per day }\right)\end{array}$ & 430 & $49 \%$ \\
\hline & $\begin{array}{c}\text { Decrease } \\
\left(<0 \mu \mathrm{g} \cdot \mathrm{mL}^{-1} \text { per day }\right)\end{array}$ & 508 & $27 \%$ \\
\hline \multirow{2}{*}{$\begin{array}{c}\text { Low } \\
\left(\geq 2.5 \mu \mathrm{g} \cdot \mathrm{mL}^{-1}\right)\end{array}$} & $\begin{array}{c}\text { Increase } \\
\left(\geq 0 \mu \mathrm{g} \cdot \mathrm{mL}^{-1} \text { per day }\right)\end{array}$ & 337 & $21 \%$ \\
\hline & $\begin{array}{c}\text { Decrease } \\
\left(<0 \mu \mathrm{g} \cdot \mathrm{mL}^{-1} \text { per day }\right)\end{array}$ & 560 & $9 \%$ \\
\hline
\end{tabular}

b)
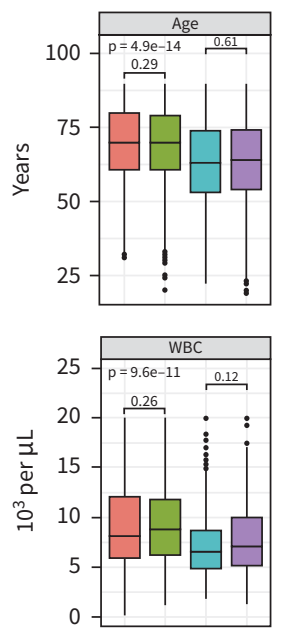
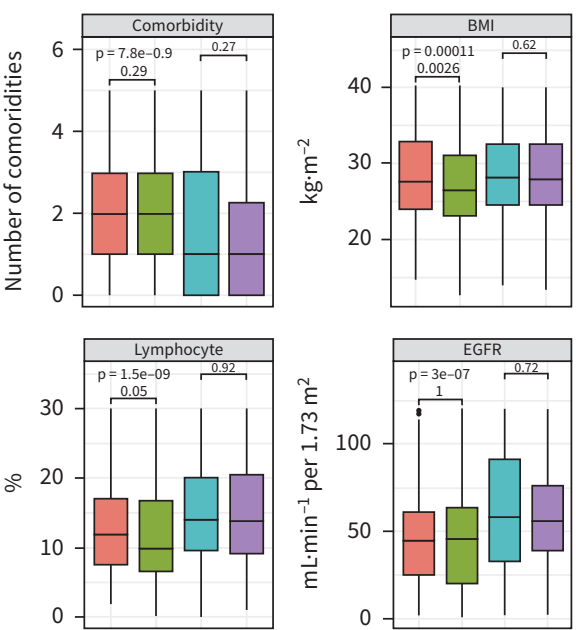

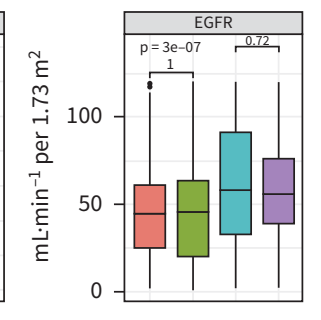

$\mathrm{HI}$
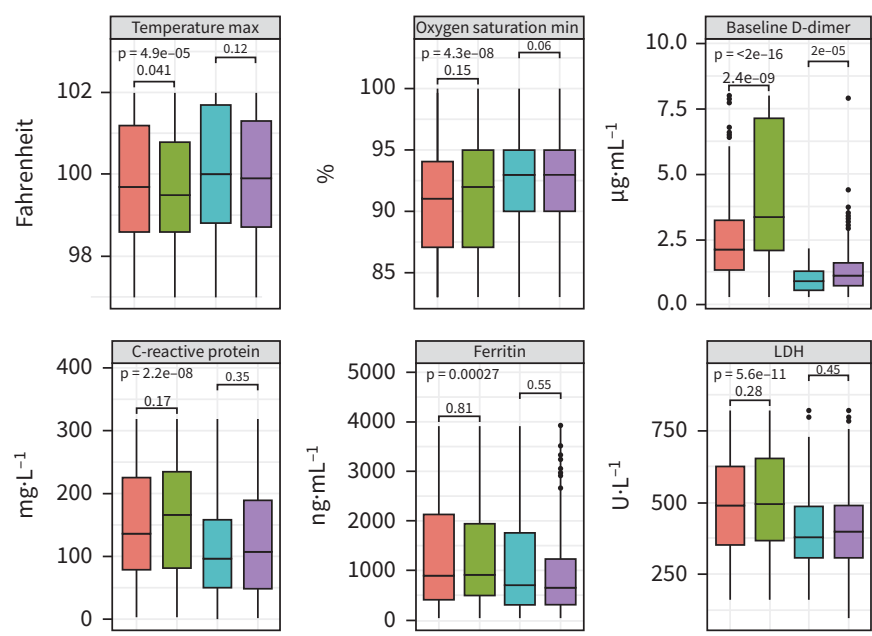

FIGURE 3 In-hospital mortality and baseline patient characteristics of four post-anticoagulation D-dimer groups. a) In-hospital mortality rates by post-anticoagulation D-dimer groups. b) Baseline characteristics of patients with different D-dimer groups after anticoagulation therapy. HI: high increase; HD: high decrease; LI: low increase; LD: low decrease; EGFR: estimated glomerular filtration rate; LDH: lactate dehydrogenase.

impact on model prediction among all clinical characteristics under consideration. Therefore, changes in D-dimer levels and trends following initiation of therapeutic doses of anticoagulation are novel prognostic biomarkers that should be considered in the management of hospitalised COVID-19 patients.

Elevated D-dimer is among the most consistent markers of poor outcomes in COVID-19 patients. Several retrospective studies of hospitalised patients in Wuhan, China, have demonstrated elevated D-dimer levels

\begin{tabular}{l|c|c|}
\cline { 2 - 3 } \multicolumn{1}{|c|}{ Varible } & OR & $95 \% \mathrm{CI}$ \\
\hline Post-A/C D-dimer HI versus LD & 6.52 & $(3.81-11.16)$ \\
\hline Post-A/C D-dimer LI versus LD & 4.06 & $(2.23-7.38)$ \\
\hline ARDS (yes versus no) & 2.46 & $(1.44-4.20)$ \\
\hline Post-A/C D-dimer HD versus LD & 2.37 & $(1.37-4.09)$ \\
\hline Acute kidney injury (yes versus no) & 1.99 & $(1.34-2.96)$ \\
\hline Potassium $\left(\mathrm{mmol}^{-1} \mathrm{~L}^{-1}\right)$ & 1.29 & $(1.03-1.60)$ \\
\hline Age (years) & 1.06 & $(1.04-1.07)$ \\
\hline LDH $\left(1000 \times \mathrm{U} \cdot \mathrm{L}^{-1}\right)$ & 1.33 & $(1.03-1.72)$ \\
\hline Platelet $\left(1000 \times \mu \mathrm{L}^{-1}\right)$ & 0.99 & $(0.99-1.00)$ \\
\hline $\mathrm{O}_{2}$ sat min $(\%)$ & 0.94 & $(0.92-0.97)$ \\
\hline
\end{tabular}
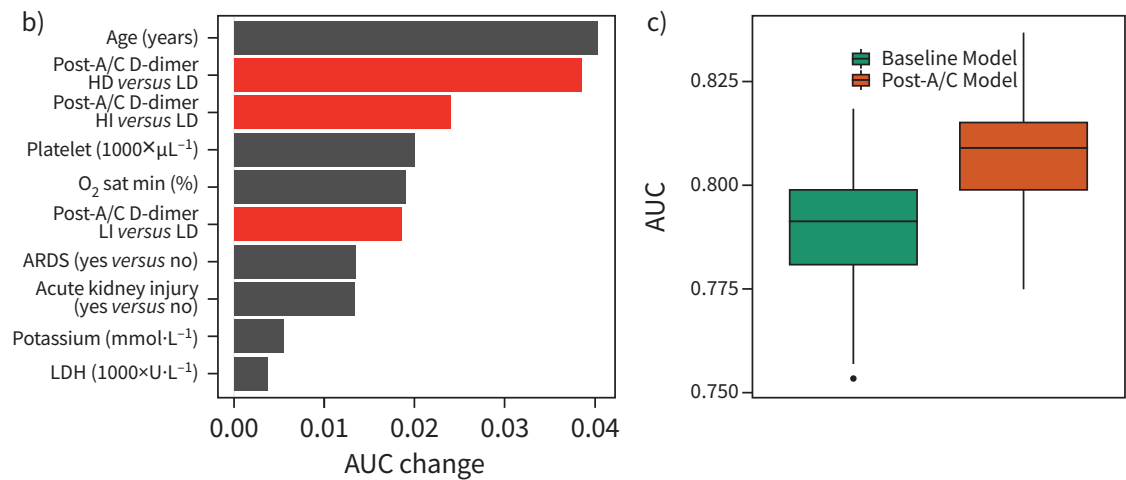

FIGURE 4 A multivariate prediction model for patients' outcome. a) ORs estimates (95\% CI), for variables selected in the post-anticoagulation model (post-A/C). b) Area under the curve (AUC) differences between leave-one-predictor-out models and the full model for variables selected in the post-anticoagulation model. c) Comparison of 10 -fold cross-validation AUCs between baseline model and post-anticoagulation model in 100 bootstrap samples. HI: high increase; HD: high decrease; LI: low increase; LD: low decrease; LDH: lactate dehydrogenase. 
on admission with differing optimal cut-offs (starting at $>0.5 \mu \mathrm{g} \cdot \mathrm{mL}^{-1}$ ) to be predictive of in-hospital mortality [3, 5, 6, 14]. However, D-dimer at the time of admission was not a significant predictor of mortality in our hospitalised cohort of COVID-19 patients with severe illness on therapeutic doses of anticoagulation for thromboprophylaxis. Instead, we found post-anticoagulant D-dimer levels to be highly predictive of in-hospital mortality in this group, with patients in the high increase group 6.58 times more likely to die during the hospitalisation than patients in the low decrease group. Interestingly, patients in the low increase post-anticoagulant D-dimer group had a higher risk of dying than those in the high decrease group, suggesting that the trend of the D-dimer following anticoagulation is more important than the 3-day mean. With limited specific baseline patient characteristics associated with post-anticoagulant D-dimer trends, it is critical that serial D-dimer measurements are collected for accurate prediction of in-hospital mortality.

D-dimer is commonly measured throughout the hospitalisation of severely ill COVID-19 patients. However, there is no consensus on how to interpret changes in D-dimer levels in this context [15]. We found that the ability of D-dimer to predict in-hospital mortality was not affected by fibrinogen levels, indicating the hypercoagulability is the result of a more COVID-specific process rather than DIC. Once on therapeutic doses of anticoagulation, elevated D-dimer levels could be expected to decrease or normalise reflecting the cessation of new clot formation. However, persistently elevated or rising D-dimer levels following anticoagulation in COVID-19 patients may signify continued risk of large vessel thrombotic events [16, 17]. In a study of 608 patients who completed anticoagulation for VTE, patients with persistently elevated D-dimer levels were 2.27-fold more likely to experience recurrent VTE than those who had normalisation of D-dimer [16]. Furthermore, recent autopsy data from COVID-19 patients demonstrated severe endothelial injury associated with intracellular virus resulting in widespread microangiopathy and pulmonary capillary microthrombi compared to matched autopsies from influenza A (H1N1) patients [18]. Therefore, D-dimer levels and trends appear to be capturing angiocentric progression associated with poor outcomes.

There are strengths and limitations to this study worth discussing. We were able to rapidly evaluate the impact of over 65 clinical features on in-hospital mortality for hospitalised COVID-19 patients with the highest risk of poor outcomes. In order to do so, we present the largest experience reporting the clinical outcomes of hospitalised COVID-19 patients receiving therapeutic doses of anticoagulation for thromboprophylaxis. However, patients treated at a single tertiary hospital network in New York City may not be representative of the general population in the USA and worldwide. Other limitations include that there may be imprecisions of laboratory assays, which can alter the assessment of D-dimer. In addition, we were unable to account for unmeasured confounders that may affect D-dimer levels, a particular limitation inherent to all observational studies.

In summary, D-dimer levels and trends should be widely incorporated into the management protocols for hospitalised COVID-19 patients on anticoagulation. We highlight an important subset of patients associated with especially poor outcomes that may benefit from early escalation to Intensive Care Unit (ICU) care and, if proven effective in this setting, emerging disease modifying treatments (i.e. remdesivir, corticosteroids) that may help reduce virus spread and/or COVID-associated hyperinflammation [19-21]. Conversely, patients in low-risk D-dimer groups following administration of therapeutic doses of anticoagulation may be appropriate for switch to prophylactic anticoagulant doses or de-escalation of care from ICU settings. Future studies should further validate changes in D-dimer following anticoagulation as a prognostic biomarker and consider D-dimer when determining the effectiveness of COVID-19 interventions in different risk groups.

Acknowledgement: We would like to express our sincerest condolences to the patients and their families who were affected by the COVID-19 pandemic. We greatly appreciate the healthcare providers who contributed to the care of these patients. The Mount Sinai deidentified COVID-19 database was supported through the computational and data resources and staff expertise provided by Scientific Computing at the Icahn School of Medicine at Mount Sinai. The authors also acknowledge the support of the Biostatistics Shared Resource Facility, Icahn School of Medicine at Mount Sinai and NCl Cancer Center Support Grant P30 CA196521-01.

Data availability: The datasets analysed during the current study are not publicly available due to US Federal Health Insurance Portability and Accountability Act compliance. A deidentified dataset may be available from the corresponding authors on reasonable request. The Icahn School of Medicine at Mount Sinai Institutional Review Board considered the study exempt. The lead author and all authors affirm that the manuscript is an honest, 
accurate, and transparent account of the study being reported; that no important aspects of the study have been omitted; and that any discrepancies from the study as planned have been explained.

Author contributions: Conception and design, manuscript writing and final approval: all authors; collection, assembly, and analysis and interpretation of data: X. Song, E. Taioli, P. Wang and R.R. Veluswamy.

Conflict of interest: None declared.

References

1 World Health Organisation. Coronavirus disease 2019 (COVID-19) Situation Report - 209. Published online November 1, 2020.

2 Artifoni M, Danic G, Gautier G, et al. Systematic assessment of venous thromboembolism in COVID-19 patients receiving thromboprophylaxis: Incidence and role of D-dimer as predictive factors. J Thromb Thrombolysis 2020; 50: 211-216.

3 Zhou F, Yu T, Du R, et al. Clinical course and risk factors for mortality of adult inpatients with COVID-19 in Wuhan, China: A retrospective cohort study. Lancet 2020; 395: 1054-1062.

4 Al-Samkari H, Karp Leaf RS, Dzik WH, et al. COVID-19 and coagulation: bleeding and thrombotic manifestations of SARS-CoV-2 infection. Blood 2020; 136: 489-500.

5 Yao Y, Cao J, Wang Q, et al. D-dimer as a biomarker for disease severity and mortality in COVID-19 patients: a case control study. J Intensive Care 2020; 8: 49.

6 Zhang L, Yan X, Fan Q, et al. D-dimer levels on admission to predict in-hospital mortality in patients with Covid-19. J Thromb Haemost 2020; 18: 1324-1329.

7 Guan WJ, Ni ZY, Hu Y, et al. Clinical characteristics of coronavirus disease 2019 in China. N Engl J Med 2020; 382: $1708-1720$

8 Chi G, Lee JJ, Jamil A, et al. Venous thromboembolism among hospitalised patients with COVID-19 undergoing thromboprophylaxis: A systematic review and meta-analysis. J Clin Med 2020; 9: 2489.

9 Yuriditsky E, Horowitz JM, Merchan C, et al. Thromboelastography profiles of critically ill patients with coronavirus disease 2019. Crit Care Med 2020; 48: 1319-1326.

10 Llitjos JF, Leclerc M, Chochois C, et al. High incidence of venous thromboembolic events in anticoagulated severe COVID-19 patients. J Thromb Haemost 2020; 18: 1743-1746.

11 Balk RA. Systemic inflammatory response syndrome (SIRS): Where did it come from and is it still relevant today? Virulence 2014; 5: 20-26.

12 Buuren S van, Groothuis-Oudshoorn K. Mice: Multivariate imputation by chained equations in R. J Stat Softw 2011; 45: 1-68.

13 Hastie T, Tibshirani R, Wainwright M. Statistical Learning with Sparsity: The Lasso and Generalisations. Boca Raton, CRC press, 2015.

14 Shah S, Shah K, Patel SB, et al. Elevated D-dimer levels are associated with increased risk of mortality in COVID-19: A systematic review and meta-analysis. Cardiol Rev 2020; 28: 295-302.

15 American Society of Hematology. COVID-19 and D-dimer: Frequently asked questions. www.hematology.org/ covid-19/covid-19-and-d-dimer

16 Palareti G, Cosmi B, Legnani C, et al. D-dimer testing to determine the duration of anticoagulation therapy. N Engl J Med 2006; 355: 1780-1789.

17 Tamizifar B, Oghab P, Esfahani MA. The prediction role of D-dimer in recurrence of venous thromboembolism 1-year after anticoagulation discontinuing following idiopathic deep vein thrombosis. J Res Med Sci Off $J$ Isfahan Univ Med Sci 2014; 19: 586-591.

18 Ackermann M, Verleden SE, Kuehnel M, et al. Pulmonary vascular endothelialitis, thrombosis, and angiogenesis in Covid-19. N Engl J Med 2020; 383: 120-128.

19 Beigel JH, Tomashek KM, Dodd LE, et al. Remdesivir for the treatment of Covid-19 - final report. N Engl J Med 2020; 383: 1813-1826.

20 Chen L, Xiong J, Bao L, et al. Convalescent plasma as a potential therapy for COVID-19. Lancet Infect Dis. 2020; 20: 398-400.

21 RECOVERY Collaborative Group, Horby P, Lim WS, et al. Dexamethasone in hospitalised patients with Covid-19 - preliminary report. N Engl J Med 2021; 384: 693-704. 